International Journal of Engineering \& Technology, $7(4.10)(2018) 694-697$
International Journal of Engineering \& Technology
SPC
Website: www.sciencepubco.com/index.php/IJET
Research paper

\title{
The Impulsive Neutral Integro-Differential Equations with Infinite Delay and Non-Instantaneous Impulses
}

\author{
V. Usha', M. Mallika Arjunan ${ }^{2}$ \\ ${ }^{1}$ Department of Mathematics, PSG College of Arts \& Science \\ Coimbatore - 641 014, Tamil Nadu, India. \\ ${ }^{2}$ Department of Mathematics, C. B. M. College, \\ Kovaipudur, Coimbatore - 641 042, Tamil Nadu, India.

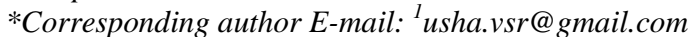

\begin{abstract}
In this manuscript, we work to accomplish the Krasnoselskii's fixed point theorem to analyze the existence results for an impulsive neutral integro-differential equations with infinite delay and non-instantaneous impulses in Banach spaces. By deploying the fixed point theorem with semigroup theory, we developed the coveted outcomes.
\end{abstract}

Keywords: Neutral equations; Equations with impulses; Non-instantaneous impulse condition; Integro-differential equations; fixed point theorem.

\section{Introduction}

In this paper, we consider the impulsive neutral integrodifferential equation with infinite delay of the model

$$
\begin{aligned}
& \frac{d}{d t}\left[w(t)-\mathrm{G}\left(\mathrm{t}, \mathrm{w}_{\mathrm{t}},\left(\mathrm{H}_{1} \mathrm{w}\right)(\mathrm{t})\right)\right]=\mathrm{Aw}(\mathrm{t})+\mathrm{G}_{2}\left(\mathrm{t}, \mathrm{w}_{\mathrm{t}},\left(\mathrm{H}_{2} \mathrm{w}\right)(\mathrm{t})\right)+ \\
& \mathrm{G}_{3}\left(\mathrm{t}, \mathrm{w}_{\mathrm{t}},\left(\mathrm{H}_{3} \mathrm{w}\right)(\mathrm{t})\right), \mathrm{t} \in\left(\mathrm{s}_{\mathrm{i}}, \mathrm{t}_{\mathrm{i}+1}\right], \mathrm{i}=0,1, \ldots \mathrm{N}, \mathrm{I}=[0, \mathrm{~T}] \\
& \mathrm{w}(\mathrm{t})=\mathrm{h}_{\mathrm{i}}\left(\mathrm{t}, \mathrm{w}_{\mathrm{t}}\right), \text { for } \mathrm{t} \in\left(\mathrm{t}_{\mathrm{i}}, \mathrm{s}_{\mathrm{i}}\right], \mathrm{i}=1,2, \ldots \mathrm{N}, \\
& \mathrm{w}_{0}=\phi \in \mathrm{B}_{\mathrm{h}},
\end{aligned}
$$

where the operator $\mathrm{A}$ is the infinitesimal generator of a analytic semigroup $\{\mathrm{T}(\mathrm{t})\}_{\mathrm{t} \geq 0}$ in a Banach space $X$ having norm $\|\cdot\|$ and $\mathrm{M}_{1}$ is a positive constant to ensure that $\|T(t)\| \leq \mathrm{M}_{1}, \mathrm{G}_{\mathrm{j}}: \mathrm{I} \times \mathrm{B}_{\mathrm{h}} \times \mathrm{X} \rightarrow$ $X, j=1,2,3$ are given $X$ - valued functions, $H_{j}, j=1,2,3$ are described as $\left(\mathrm{H}_{\mathrm{j}} \mathrm{w}\right)(\mathrm{t})=\int_{0}^{t} e_{\mathrm{j}}\left(\mathrm{t}, \mathrm{s}, \mathrm{w}_{\mathrm{t}}\right)$ ds where $\mathrm{e}_{\mathrm{j}}: \mathrm{D} \times \mathrm{B}_{\mathrm{h}} \rightarrow \mathrm{X}, \mathrm{j}=$ $1,2,3 ; \mathrm{D}=\{(\mathrm{t}, \mathrm{s}) \in \mathrm{I} \times \mathrm{I}: 0 \leq \mathrm{s} \leq \mathrm{t} \leq \mathrm{T}\}$ are suitable functions, and $B_{h}$ is a phase space characterized in preliminaries. Here $0=t_{0}<t_{1}$ $<\mathrm{t}_{2}<\cdots<\mathrm{t}_{\mathrm{m}}<\mathrm{t}_{\mathrm{m}+1}=\mathrm{T}$. For any continuous function $\mathrm{w}$ indicated on $(-\infty, T]$ and any $t \geq 0$, we represent by $w_{t}$ the part of $B_{h}$ specified by $\mathrm{w}_{\mathrm{t}}(\theta)=\mathrm{w}(\mathrm{t}+\theta)$ for $\theta \in(-\infty, 0]$. The following $\mathrm{w}_{\mathrm{t}}(\cdot)$ denote the history of the state from time $-\infty$, up to the current time t.

The study of abstract differential equations with non-instantaneous impulses was initiated recently by Hernández and O'Regan in [1]. Lately, M. Pierri, D. O'Regan and V. Rolnik [3] study the existence results of some abstract differential equations with noninstantaneous impulses with the help of fractional powers of operators and semigroup theory.

Our objective here is to give existence results for the given problem by using semigroup theory. In section 2 , we recall some preliminary results and definitions which will be utilized throughout this manuscript. In section 3 , we present and prove the existence of solutions for the given problem. Our approach here is based on Krasnoselskii's fixed point theorem. Finally in section 4, an application is given to demonstrate the gained results.

\section{Preliminaries}

Let $\mathrm{X}$ be a Banach space provided with the norm $\|\cdot\|$. Consider the analytic semigroup $\{\mathrm{T}(\mathrm{t})\}_{\mathrm{t} \geq 0}$ bounded linear operators in $\mathrm{X}$. Let $\mathrm{A}$ : $\mathrm{D}(\mathrm{A}) \rightarrow \mathrm{X}$ be the infinitesimal generator of $\{\mathrm{T}(\mathrm{t})\}_{\mathrm{t} \geq 0}$. Then it is possible to determine the fractional power $\mathrm{A}^{\alpha}$ for $0<\alpha \leq 1$, as a closed linear operator on its domain $\mathrm{D}(\mathrm{A})^{\alpha}$, being dense in $\mathrm{X}$. If $\mathrm{X}^{\alpha}$ represents the space $\mathrm{D}(\mathrm{A})^{\alpha}$ endowed with the norm

$$
\|x\|^{\alpha}=\left\|A^{\alpha} \mathrm{x}\right\|, \mathrm{x} \in \mathrm{D}\left(\mathrm{A}^{\alpha}\right) .
$$

Then the following properties are well known. With this discussion, we recall fundamental properties of fractional powers $A^{\alpha}$ from Pazy [2]. Now we consider the space

$B_{h}^{\prime}=\mathrm{PC}((-\infty, \mathrm{T}), \mathrm{X})=\left\{\mathrm{w}:(-\infty, \mathrm{T}] \rightarrow \mathrm{X}\right.$ such that $\mathrm{w}_{\mathrm{k}} \in \mathrm{C}\left(\mathrm{I}_{\mathrm{k}}, \mathrm{X}\right)$ and there exists $w\left(t_{k}^{+}\right)$and $\left.w\left(t_{k}^{-}\right), w_{0}=\emptyset \in B_{h}, k=0,1,2, \ldots m\right\}$ Where $\mathrm{w}_{\mathrm{k}}$ is the restriction of $\mathrm{w}$ to $\mathrm{I}_{\mathrm{k}}=\left(t_{k}, t_{k+1}\right]$, set $\|\cdot\|_{B_{h}^{\prime}}$ be the seminorm in $B_{h}^{\prime}$ defined by $\|w\|_{B_{h}^{\prime}}=\|\phi\|_{\mathrm{B}_{\mathrm{h}}}+\sup \{|\mathrm{w}(\mathrm{s})|: \mathrm{S} \in[0, \mathrm{~T}]\}$ , w $\in B_{h}^{\prime}$.

\section{Existence results}

Definition 3.1: A function w: $(-\infty, \mathrm{T}] \longrightarrow \mathrm{X}$ is called mild solution of model (1)-(3) if $\boldsymbol{w}_{\mathbf{0}}=\boldsymbol{\phi} \in \mathrm{Bh}$ on $(-\infty, 0] \mathrm{w}(\mathrm{t})=\boldsymbol{h}_{\boldsymbol{i}}\left(\boldsymbol{t}, \boldsymbol{w}_{\boldsymbol{t}}\right)$ for $\mathrm{t} \in$ (ti , si] and for each $\mathrm{i}=0,1, \ldots \mathrm{N}$, the constraint of $\mathrm{w}($.$) to interval$ $(0, \mathrm{~T}]-\left\{\boldsymbol{t}_{\mathbf{1}}, \boldsymbol{t}_{\mathbf{2}}, \boldsymbol{t}_{\mathbf{3}}, \boldsymbol{t}_{\mathbf{4}}, \ldots . \boldsymbol{t}_{\boldsymbol{m}}\right\}$ is continuous and for each $\mathrm{s} \in[0, \mathrm{~T})$ the function $\mathrm{AT}(\mathrm{t}-\mathrm{s}) \boldsymbol{G}_{\mathbf{1}}\left(\mathrm{s}, \boldsymbol{w}_{\boldsymbol{s}}, \int_{\mathbf{0}}^{\boldsymbol{t}} \boldsymbol{e}_{\mathbf{1}}\left(\boldsymbol{s}, \boldsymbol{\tau}, \boldsymbol{w}_{\boldsymbol{\tau}}\right) \boldsymbol{d} \boldsymbol{\tau}\right)$ is integrable and sub-sequent impulsive integral equation is 


$$
\begin{aligned}
& \int \mathbf{T}(\mathbf{t})\left[\phi(0)-\mathbf{G}_{1}(\mathbf{0}, \boldsymbol{\phi}, \mathbf{0})+\xi_{1}+\int_{0}^{\mathrm{t}} \mathrm{A} \xi_{2} \mathrm{~d} s\right. \\
& +\int_{0}^{\mathrm{t}} \xi_{3} \mathrm{~d} s+\int_{0}^{\mathrm{t}} \xi_{4} \mathrm{ds}, \mathrm{t} \in\left[0, \mathbf{t}_{1}\right] \\
& \mathbf{W}(\mathbf{t})=\left\{\begin{array}{c}
\mathbf{h}_{\mathbf{i}}\left(\mathbf{t}, \mathbf{w}_{\mathbf{t}}\right), \mathbf{t} \in\left(\mathbf{t}_{\mathbf{i}}, \mathbf{s}_{\mathbf{i}}\right] \text { for } \mathbf{i}=\mathbf{1}, 2,3, \ldots, \mathbf{N} . \\
\mathbf{T}\left(\mathbf{t}-\mathbf{s}_{\mathbf{i}}\right)\left[\mathbf{h}\left(\mathbf{s}_{\mathbf{i}}, \mathbf{w}_{\mathbf{i}}\right)-\xi_{5}\right]+\xi_{1}+\int_{s_{i}}^{t} A \xi_{2} d s
\end{array}\right. \\
& +\int_{s_{i}}^{t} \xi_{3} d s+\int_{s_{i}}^{t} \xi_{4} d s t \in\left(s_{i}, t_{i+1},\right]
\end{aligned}
$$

Where $\boldsymbol{\xi}_{\mathbf{j}}=\mathbf{T}(\mathbf{t}-\mathbf{s}) \mathbf{G}_{\mathbf{k}}\left(\mathbf{s}, \mathbf{w}_{\mathbf{s}}, \int_{0}^{\mathbf{s}} \mathbf{e}_{\mathbf{k}}\left(\mathbf{t}, \mathbf{s}, \mathbf{w}_{\mathbf{s}}\right)\right) \mathbf{d} \mathbf{s}$ for $\mathrm{j}=2,3,4$ and $\mathrm{k}=1,2,3, \quad \xi_{\mathbf{1}}=\mathbf{G}_{\mathbf{1}}\left(\mathrm{t}, \mathbf{w}_{\mathrm{t}}, \int_{\mathbf{0}}^{\mathrm{t}} \mathbf{e}_{\mathbf{1}}\left(\mathbf{t}, \mathbf{s}, \mathbf{w}_{\mathbf{s}}\right) \mathbf{d s}\right)$ and $\boldsymbol{\xi}_{\mathbf{5}}=\mathbf{G}_{\mathbf{1}}\left(\mathbf{s}_{\mathrm{i}}, \mathbf{w}_{\mathbf{s}_{\mathbf{i}}}\right.$ $\left.\int_{0}^{s_{i}} \mathbf{e}_{1}\left(s_{i}, \tau, \mathbf{w}_{\tau}\right)\right) d \tau$.

In order to study the existence results for the problem (1)- (3) we need to list the following hypotheses:

(H1) For the infinitesimal generator A of a compact analytic semi-group $0 \in \mathbf{Q}(\mathrm{A})$ there exist conststants $\mathbf{M}_{\mathbf{1}}$ and $\mathbf{M}_{\mathbf{0}}$ such that $\|\mathbf{T}(\mathrm{t})\|_{\mathrm{L}(\mathrm{X})} \leq \mathbf{M}_{\mathbf{1}}$ for all $\mathrm{t} \geq \mathbf{0}, \mathbf{M}_{\mathbf{0}}=\left\|\mathrm{A}^{-\boldsymbol{\beta}}\right\|$, and $\left\|(\mathbf{A})^{1-\beta} \mathbf{T}(\mathbf{t}, \mathbf{s})\right\| \leq \frac{\mathbf{M}_{1-\beta}}{(\mathbf{t}-\mathbf{s})^{1-\beta}}, 0 \leq \mathbf{t} \leq \mathbf{T}$.

(H2) For the continuous function $\mathbf{G}_{\mathbf{1}}: \mathrm{I} \times \mathbf{B}_{\mathbf{h}} \times \mathbf{X} \rightarrow \mathbf{X}$ there exist a positive constant $\boldsymbol{\beta} \boldsymbol{\epsilon}(\mathbf{0}, \mathbf{1}), \mathbf{K}_{\mathbf{G}_{1}}>\mathbf{0}, \widehat{\mathbf{K}_{\mathbf{G}}}>\mathbf{0}$ and $\mathbf{K}_{\mathbf{G}_{1}}^{*}>\mathbf{0}$ such that $\mathbf{G}_{\mathbf{1}}$ is $\mathbf{X}_{\boldsymbol{\beta}^{-}}$valued and for all $\left(\mathrm{t}, \boldsymbol{\varphi}_{\mathbf{j}}\right) \in \mathrm{I} \times \mathbf{B}_{\mathbf{h}}, \mathrm{x}, \mathrm{y} \in \mathrm{X}, \mathrm{j}=1,2$; (i) $\left\|A^{\beta} G_{1}\left(t, \varphi_{1}, \mathbf{x}\right)-A^{\beta} G_{1}\left(t, \varphi_{2}, y\right)\right\|_{x} \leq K_{G_{1}}\left\|\varphi_{1}-\varphi_{2}\right\|_{B_{h}}+$ $\widehat{\mathbf{K}}_{\mathbf{G}}\|\mathbf{x}-\mathbf{y}\|_{\mathbf{X}}, \mathrm{X}, \mathrm{y} \in \mathrm{X}$

(ii) $\left\|A^{\beta} G_{1}(t, \varphi, 0)\right\|_{x} \leq K_{G_{1}}\|\varphi\|_{B_{h}}+K_{G_{1}}^{*}$ and $K_{G_{1}}^{*}=$ $\max _{t \in I}\left\|A^{\beta} G_{1}(t, 0,0)\right\|_{x}$.

(H3) For the continuous function $\mathbf{G}_{\mathbf{2}}: \mathrm{I} \times \mathbf{B}_{\mathbf{h}} \times \mathbf{X} \rightarrow \mathbf{X}$ is strongly measurable and we can find $\mathbf{m}_{\mathbf{G}_{2}}:[0, \mathrm{~T}] \rightarrow(\mathbf{0}, \infty)$ and a nondecreasing function $\boldsymbol{\Omega}_{\mathbf{G}_{\mathbf{2}}}:[0, \infty) \rightarrow(\mathbf{0}, \infty)$ such that for all $\left(\mathrm{t}, \boldsymbol{\varphi}_{\mathbf{j}}\right)$ $\in I \times B_{h}, \quad j=1,2 ; \quad x \quad$ in $X,\left\|G_{2}(t, \varphi, x)\right\|_{x} \leq \mathbf{m}_{G_{2}}(t) \Omega_{G_{2}}(\|\varphi\|+$ $\|\mathbf{x}\|)_{\mathbf{B}_{\mathbf{h}}}$.

(H4) There exists positive constants $\mathbf{K}_{\mathbf{G}_{3}}>\mathbf{0}, \widehat{\mathbf{K}_{\mathbf{G}_{3}}}>\mathbf{0}$ and $\mathbf{K}_{\mathbf{G}_{3}}^{*}>\mathbf{0}$ and the continuous function $\mathbf{G}_{\mathbf{3}}$ : $\mathbf{I} \times \mathbf{B}_{\mathbf{h}} \times \mathbf{X} \rightarrow \mathbf{X}$ for all ( $\left.\mathrm{t}, \quad \boldsymbol{\varphi}_{\mathbf{j}}\right) \in \mathrm{I} \times \mathbf{B}_{\mathbf{h}}, \quad \mathrm{j}=1,2 ;$ such that (i) $\left\|G_{3}\left(t, \varphi_{1}, \mathbf{x}\right)-G_{3}\left(t, \varphi_{2}, y\right)\right\|_{x} \leq K_{G_{3}}\left\|\varphi_{1}-\varphi_{2}\right\|_{B_{h}}+\widehat{K_{G_{3}}} \| x-$ $\mathbf{y} \|_{\mathbf{x}}$ $\mathrm{x}, \mathrm{y}$ $\epsilon$ (ii) $\left\|A^{\beta} G_{3}(t, \varphi, 0)\right\|_{x} \leq K_{G_{3}}\|\varphi\|_{B_{h}}+K_{G 1}^{*}$ and $K_{G_{3}}^{*}=$ $\max _{t \in I}\left\|G_{1}(t, 0,0)\right\|_{x}$.

(H5)

$K_{h_{i}}>0$, which is considered as a constant and $I=$

For

$\mathbf{1}, 2, \ldots, \mathbf{N}$, such that $\left\|\mathbf{h}_{\mathbf{i}}\left(\mathbf{t}, \varphi_{1}\right)-\mathbf{h}_{\mathbf{i}}\left(\mathbf{t}, \boldsymbol{\varphi}_{2}\right)\right\|_{\mathbf{x}} \leq \mathbf{K}_{\mathbf{h}_{\mathbf{I}}} \| \varphi_{1}-$ $\boldsymbol{\varphi}_{2} \|_{\mathrm{B}_{\mathrm{h}}}$, for each $\mathrm{t} \in\left(\mathbf{t}_{\mathrm{I}}, \mathbf{s}_{\mathbf{i}}\right]$ and all $\boldsymbol{\varphi}_{1}, \boldsymbol{\varphi}_{2} \in \mathrm{B}_{\mathbf{h}}$.

(H6) The functions $\mathbf{e}_{\mathbf{j}}: \mathfrak{D} \times \mathbf{B}_{\mathbf{h}} \rightarrow \mathbf{X}$ are continuous and there exist constants $\mathbf{K}_{\mathbf{e}_{\mathbf{j}}}>\mathbf{0}, \quad \mathbf{K}_{\mathbf{e}_{\mathbf{j}}}^{*}>\mathbf{0}$ to ensure that $\| \mathbf{e}_{\mathbf{j}}(\mathbf{t}, \mathbf{s}, \boldsymbol{\varphi})-$ $\mathbf{e}_{\mathbf{j}}(\mathbf{t}, \mathbf{s}, \Psi)\left\|_{\mathrm{x}} \leq \mathbf{K}_{\mathrm{e}_{\mathrm{j}}}\right\| \boldsymbol{\varphi}-\boldsymbol{\Psi} \|_{\mathbf{B}_{\mathrm{h}}},(\mathrm{t}, \mathrm{s}) \in \mathfrak{D}, \boldsymbol{\varphi}, \boldsymbol{\Psi} \in \mathbf{B}_{\mathbf{h}}^{2} ; \quad$ and $\quad \mathbf{K}_{\mathbf{e}_{\mathbf{j}}}^{*}=$ $\max _{\mathbf{t} \in \mathbf{I}}\left\|\mathbf{e}_{\mathbf{j}}(\mathbf{t}, \mathbf{s}, \mathbf{0})\right\|_{\mathbf{x}}, \mathbf{j}=1,2,3$.
(H7) The following inequalities holds: Let $\mathrm{M}_{1}\left[\mathrm{~K}_{\mathrm{h}_{\mathrm{i}}}\left(\mathrm{D}_{1}^{*} \mathrm{q}+\right.\right.$ $\left.\left.\mathrm{c}_{\mathrm{n}}\right)+\mathrm{K}_{\mathrm{h}_{\mathrm{i}}}^{*}\right]+\left(\mathrm{M}_{0}\left(1+\mathrm{M}_{1}\right)+\frac{\mathrm{M}_{1}-\beta^{\mathrm{T}^{\beta}}}{\beta}\left[\left(\mathrm{K}_{\mathrm{G}_{1}}+\widehat{\mathrm{K}_{\mathrm{G}_{1}}} \mathrm{TK}_{\mathrm{e}_{1}}\right)\right.\right.$

$\mathrm{D}_{1}^{*} \mathrm{q}+\mathrm{p}_{1} \mathrm{M}_{1} T \mathrm{~m}_{\mathrm{G}_{2}}(\mathrm{~s}) \Omega_{\mathrm{G}_{2}}\left[\left(1+\mathrm{TK}_{\mathrm{e}_{2}}\right) \mathrm{D}_{1}^{*} \mathrm{q}+\mathrm{p}_{2}\right]+\mathrm{M}_{0} \mathrm{M}_{1} \mathrm{~T}\left[\left(\mathrm{~K}_{\mathrm{G}_{3}}+\right.\right.$

$\left.\left.\mathrm{K}_{\mathrm{G}_{3}} \mathrm{~T} \mathrm{~K} \mathrm{~K}_{\mathrm{e}_{3}}\right) \mathrm{D}_{1}^{*} \mathrm{q}+\mathrm{p}_{3}\right] \leq \mathrm{q}, \mathrm{t} \in[0, \mathrm{~T}]$ where

$$
\begin{aligned}
& \mathbf{p}_{\mathbf{1}}=\left[\left(\mathbf{K}_{\mathbf{G}_{1}}+\widehat{\mathbf{K}_{\mathbf{G}_{1}}} \mathrm{TK_{ \mathbf {e } _ { 1 } }}\right) \mathbf{c}_{\mathbf{n}}+\widehat{\mathbf{K}_{\mathbf{G}_{1}}} \mathrm{TK}_{\mathbf{e}_{1}}^{*}+\mathbf{K}_{\mathbf{G}_{1}}^{*}\right], \\
& \mathbf{p}_{2}=\mathbf{c}_{\mathbf{n}}+\mathbf{T K}_{\mathbf{e}_{2}} \mathbf{c}_{\mathbf{n}}+\mathrm{TK}_{\mathbf{e}_{2}}^{*} \\
& \mathbf{p}_{3}=\left[\left(\mathbf{K}_{\mathbf{G}_{3}}+\widehat{\mathbf{K}_{\mathbf{G}_{3}}} \mathrm{~T} \mathbf{K}_{\mathbf{e}_{3}}\right) \mathbf{c}_{\mathbf{n}}+\widehat{\mathbf{K}_{\mathbf{G}_{3}}} \mathrm{~T} \mathbf{K}_{\mathbf{e}_{3}}^{*}+\mathbf{K}_{\mathbf{G}_{3}}^{*}\right] .
\end{aligned}
$$

Theorem 3.1. Suppose that the hypotheses (H1)-(H7) hold. Then the structure (1)-(3) has a unique solution on I. Then

$$
\begin{aligned}
& A^{*}=D_{1}^{*}\left\{M_{1} K_{h_{i}}+\left(\left(1+M_{1}\right) M_{0}+\frac{M_{1-\beta} T^{\beta}}{\beta}\right)\left[K_{G_{1}}+\widetilde{K}_{G_{1}} T_{e_{1}}\right]+\right. \\
& \left.M_{1} D_{1}^{*}\left[K_{G_{3}}+\widetilde{K}_{G_{3}} T_{e_{3}}\right]\right\}<1 .
\end{aligned}
$$

Proof:

The problems (1)-(3) will be transformed into a fixed point problem. Consider the operator $\Phi: \mathrm{B}_{\mathrm{h}}^{\prime} \rightarrow \mathrm{B}_{\mathrm{h}}^{\prime}$ by

$$
=\left\{\begin{array}{c}
\mathrm{T}(\mathrm{t})\left[\varphi(0)-\mathrm{G}_{1}(0, \varphi, 0)\right]+\xi_{1}+\int_{0}^{\mathrm{t}} \mathrm{A} \xi_{2} \mathrm{ds}+\int_{0}^{\mathrm{t}} \xi_{3} \mathrm{ds} \\
+\int_{0}^{\mathrm{t}} \xi_{4} \mathrm{ds}, \text { for } \mathrm{t} \in\left[0, \mathrm{t}_{1}\right], \\
\mathrm{h}\left(\mathrm{t}, \mathrm{w}_{\mathrm{t}}\right), \mathrm{t} \in\left(\mathrm{t}_{\mathrm{i}}, \mathrm{s}_{\mathrm{i}}\right], \text { for } \mathrm{i}=1,2, \ldots, \mathrm{N}, \\
\mathrm{T}\left(\mathrm{t}-\mathrm{s}_{\mathrm{i}}\right)\left[\mathrm{h}_{\mathrm{i}}\left(\mathrm{s}_{\mathrm{i}}, \mathrm{w}_{\mathrm{s}_{\mathrm{i}}}\right)-\xi_{5}\right]+\xi_{1}+\int_{\mathrm{s}_{\mathrm{i}}}^{\mathrm{t}} \mathrm{A} \xi_{2} \mathrm{ds}+\int_{\mathrm{s}_{\mathrm{i}}}^{\mathrm{t}} \xi_{3} \mathrm{ds} \\
+\int_{\mathrm{s}_{\mathrm{i}}}^{\mathrm{t}} \xi_{4} \mathrm{ds}, \text { for } \mathrm{t} \in\left[\mathrm{s}_{\mathrm{i}}, \mathrm{t}_{\mathrm{i}+1}\right] .
\end{array}\right.
$$

Obviously the fixed points of the operator $\Phi$ are mild solutions of the model (1)-(3).

If $w($.$) fulfills (5), we can easily split it as w(t)=u(t)+v(t)$, for all $t \in I$, this means $w_{t}=u_{t}+v_{t}$. Let $B_{h}^{\prime \prime}=\left\{u \in B_{h}^{\prime}: u_{0}=\right.$ $\left.0 \in \mathrm{B}_{\mathrm{h}}\right\}$. Let $\|\cdot\|_{\mathrm{B}_{\mathrm{h}}^{\prime \prime}}$ be the seminorm in $\mathrm{B}_{\mathrm{h}}^{\prime \prime}$ described by $\|\mathrm{u}\|_{\mathrm{B}_{\mathrm{h}}^{\prime \prime}}=\sup _{\mathrm{t} \in \mathrm{I}}\|\mathrm{u}(\mathrm{t})\|_{\mathrm{X}}+\left\|\mathrm{u}_{0}\right\|_{\mathrm{B}_{\mathrm{h}}}=\sup _{\mathrm{t} \in \mathrm{I}}\|\mathrm{u}(\mathrm{t})\|_{\mathrm{X}}, \mathrm{u} \in \mathrm{B}_{\mathrm{h}}^{\prime \prime} \quad$ as a result $\left(B_{h}^{\prime \prime},\|\cdot\|_{B_{h}^{\prime \prime}}\right)$ is a banach space.

Consider $\mathrm{B}_{\mathrm{q}}=\left\{\mathrm{u} \in \mathrm{B}_{\mathrm{h}}^{\prime \prime}:\|\mathrm{u}\|_{\mathrm{X}} \leq \mathrm{q}\right\}$ for some $\mathrm{q} \geq 0$; then for each $\mathrm{q}, \quad \mathrm{B}_{\mathrm{q}} \subset \mathrm{B}_{\mathrm{h}}^{\prime \prime}$ is clearly bounded closed convex set. For $\mathrm{u} \in \mathrm{B}_{\mathrm{q}},\left\|\mathrm{u}_{\mathrm{s}}+\mathrm{v}_{\mathrm{s}}\right\|_{\mathrm{B}_{\mathrm{h}}} \leq \mathrm{D}_{1}^{*}\|\mathrm{u}\|_{\mathrm{X}}+\left(\mathrm{D}_{1}^{*} \mathrm{M}_{1}+\mathrm{D}_{2}^{*}\right)\|\varphi\|_{\mathrm{B}_{\mathrm{h}}}$.

In the event that $\|\mathrm{u}\|_{\mathrm{X}}<q, q>0$ then

$\left\|\mathrm{u}_{\mathrm{s}}+\mathrm{v}_{\mathrm{s}}\right\|_{\mathrm{B}_{\mathrm{h}}} \leq \mathrm{D}_{1}^{*}+\mathrm{c}_{\mathrm{n}}$

where $c_{n}=\left(D_{1}^{*} M_{1}+D_{2}^{*}\right)\|\varphi\|_{B_{h}}$. We introduce the operator $\bar{\Phi}: \mathrm{B}_{\mathrm{h}}^{\prime \prime} \rightarrow \mathrm{B}_{\mathrm{h}}^{\prime \prime}$ where $\bar{\Phi}$ maps $\mathrm{B}_{\mathrm{q}}\left(0, \mathrm{~B}_{\mathrm{h}}^{\prime \prime}\right)$ into $\mathrm{B}_{\mathrm{q}}\left(0, \mathrm{~B}_{\mathrm{h}}^{\prime \prime}\right)$. For any $\mathrm{u}(.) \in \mathrm{B}_{\mathrm{h}}^{\prime \prime}$,

$$
\begin{aligned}
& (\bar{\Phi} \mathrm{u})(\mathrm{t})=\left\{\begin{array}{c}
-\mathrm{T}(\mathrm{t}) \mathrm{G}_{1}(0, \varphi, 0)+\xi_{1}+\int_{0}^{\mathrm{t}} \mathrm{A} \xi_{2} \mathrm{ds}+\int_{0}^{\mathrm{t}} \xi_{3} \mathrm{ds}+ \\
\int_{0}^{\mathrm{t}} \xi_{4} \mathrm{ds}, \text { for } \mathrm{t} \in\left[0, \mathrm{t}_{1}\right], \\
\mathrm{h}_{\mathrm{i}}\left(\mathrm{t}, \mathrm{u}_{\mathrm{t}}+\mathrm{v}_{\mathrm{t}}\right), \mathrm{t} \in\left(\mathrm{t}_{\mathrm{i}}, \mathrm{s}_{\mathrm{i}}\right], \text { fori }=1,2, \ldots, \mathrm{N}, \\
\mathrm{T}\left(\mathrm{t}-\mathrm{s}_{\mathrm{i}}\right)\left[\mathrm{h}_{\mathrm{i}}\left(\mathrm{s}_{\mathrm{i}}, \mathrm{u}_{\mathrm{s}_{\mathrm{i}}}+\mathrm{v}_{\mathrm{s}_{\mathrm{i}}}\right)-\xi_{5}\right]+\xi_{1}+\int_{\mathrm{s}_{\mathrm{i}}}^{\mathrm{t}} \mathrm{A} \xi_{2} \mathrm{ds}+ \\
\mathrm{t} \xi_{\mathrm{s}_{\mathrm{i}}}^{\mathrm{t}} \mathrm{ds}+\int_{\mathrm{s}_{\mathrm{i}}}^{\mathrm{t}} \xi_{4} \mathrm{ds}, \text { for } \mathrm{t} \in\left[\mathrm{s}_{\mathrm{i}}, \mathrm{t}_{\mathrm{i}+1}\right],
\end{array}\right. \\
& \text { Where } \xi_{1}=\mathrm{G}_{1}\left(\mathrm{t}, \mathrm{u}_{\mathrm{t}}+\mathrm{v}_{\mathrm{t}}, \int_{0}^{\mathrm{t}} \mathrm{e}_{1}\left(\mathrm{t}, \mathrm{s}, \mathrm{u}_{\mathrm{s}}+\mathrm{v}_{\mathrm{s}}\right) \mathrm{ds}\right),
\end{aligned}
$$


$\xi_{3}=\mathrm{T}(\mathrm{t}-\mathrm{s}) \mathrm{G}_{2}\left(\mathrm{~s}, \mathrm{u}_{\mathrm{s}}+\mathrm{v}_{\mathrm{s}}, \int_{0}^{\mathrm{s}} \mathrm{e}_{1}\left(\mathrm{~s}, \tau, \mathrm{u}_{\tau}+\mathrm{v}_{\tau}\right) \mathrm{d} \tau\right)$,

$\xi_{4}=\mathrm{T}(\mathrm{t}-\mathrm{s}) \mathrm{G}_{3}\left(\mathrm{~s}, \mathrm{u}_{\mathrm{s}}+\mathrm{v}_{\mathrm{s}}, \int_{0}^{\mathrm{s}} \mathrm{e}_{3}\left(\mathrm{~s}, \tau, \mathrm{u}_{\tau}+\mathrm{v}_{\tau}\right) \mathrm{d} \tau\right)$ and

$\xi_{5}=G_{1}\left(s_{i}, u_{s_{i}}+v_{s_{i}}, \int_{0}^{s_{i}} e_{1}\left(s_{i}, \tau, u_{\tau}+v_{\tau}\right) d \tau\right)$. From this, it is understood that the operator $\Phi$ has a fixed point if and only if $\bar{\Phi}$ has a fixed point. Give us a chance to demonstrate that $\bar{\Phi}$ has a fixed point. Now, we enter the main proof of the theorem. To apply Krasnoselskii's fixed point theorem, we introduce the decomposition

$\bar{\Phi}=\sum_{\mathrm{i}=0}^{\mathrm{N}} \Phi_{\mathrm{i}}^{1}+\sum_{\mathrm{i}=0}^{\mathrm{N}} \Phi_{\mathrm{i}}^{2}$

$$
\begin{gathered}
\left(\Phi_{i}^{1} u\right)(t)=\left\{\begin{array}{c}
-T(t)\left\{G_{1}(0, \Phi, 0)+\xi_{1}+\int_{0}^{t} \xi_{2} d s+\int_{0}^{t} \xi_{4} d s,\right. \\
\text { for } t \in\left[0, t_{1}\right], \\
0, \quad \text { for } t \in\left[t_{i}, t_{i+1}\right], \quad i \geq 0 \\
h_{i}\left(t, u_{t}+v_{t}\right), \text { for } t \in\left(t_{i}, s_{i}\right], i=1,2, \ldots, N, \\
T\left(t-s_{i}\right)\left[h_{i}\left(s_{i}, u_{s_{i}}+v_{s_{i}}\right)-\xi_{5}\right]+\xi_{1}+\int_{s_{i}}^{t} \xi_{2} d s
\end{array}\right. \\
\left(\Phi_{i}^{2} u\right)(t)=\left\{\begin{array}{c}
t \\
\quad \int_{s_{i}}^{t} \xi_{4} d s, \\
\text { for } t \in\left[s_{i}, t_{i+1}\right], i=1,2, \ldots, N . \\
\int_{0}^{t} \xi_{3} d s, t \in\left[0, t_{1}\right], \\
0, \text { for } t \in\left(t_{i}, s_{i}\right],
\end{array}\right. \\
\int_{s_{i}}^{t} \xi_{3} d s, \text { for } t \in\left[s_{i}, t_{i+1}\right], i=1,2, \ldots, N .
\end{gathered}
$$

For better readability, we divide our results into four steps.

Step 1: First we show that $\Phi_{\mathrm{i}}^{1} \mathrm{u}(\mathrm{t})+\Phi_{\mathrm{i}}^{2} \mathrm{u}(\mathrm{t}) \in \mathrm{B}_{\mathrm{q}}$,

whenever $\mathrm{u} \in \mathrm{B}_{\mathrm{q}}$. For all $\mathrm{u} \in \mathrm{B}_{\mathrm{q}}$, we have

(i) $\left\|\Phi_{\mathrm{i}}^{1} \mathrm{u}(\mathrm{t})+\Phi_{\mathrm{i}}^{2} \mathrm{u}(\mathrm{t})\right\| \leq \mathrm{M}_{1} \mathrm{M}_{0}\left[\mathrm{~K}_{\mathrm{G}_{1}}\|\varphi\|_{\mathrm{B}_{\mathrm{h}}}+\mathrm{K}_{\mathrm{G}_{1}}^{*}\right]+$ $\left[\mathrm{M}_{0}+\frac{\mathrm{M}_{1-\beta} \mathrm{T}^{\beta}}{\beta}\right]\left[\left(\mathrm{K}_{\mathrm{G}_{1}}+\widetilde{\mathrm{K}}_{\mathrm{G}_{1}} \mathrm{TKe}_{1}\right) \mathrm{D}_{1}^{*} \mathrm{q}+\mathrm{p}_{1}\right]+$ $\mathrm{M}_{0} \mathrm{M}_{1} \mathrm{~T}\left[\left(\mathrm{~K}_{\mathrm{G}_{3}}+\widetilde{\mathrm{K}}_{\mathrm{G}_{3}} \mathrm{~T} \mathrm{Ke}_{3}\right) \mathrm{D}_{1}^{*} \mathrm{q}+\mathrm{p}_{3}\right]+\mathrm{M}_{1} \mathrm{Tm}_{\mathrm{G}_{2}}(\mathrm{~s}) \Omega_{\mathrm{G}_{2}}$ $\left[\left(1+\mathrm{TKe}_{2}\right) \mathrm{D}_{1}^{*} \mathrm{q}+\mathrm{p}_{2}\right] \leq \mathrm{q}, \mathrm{t} \in\left[0, \mathrm{t}_{1}\right]$,

(ii) $\left\|\Phi_{\mathrm{i}}^{1} \mathrm{u}(\mathrm{t})+\Phi_{\mathrm{i}}^{2} \mathrm{u}(\mathrm{t})\right\| \leq \mathrm{K}_{\mathrm{h}_{\mathrm{i}}}\left(\mathrm{D}_{1}^{*} \mathrm{q}+\mathrm{c}_{\mathrm{n}}\right)+\mathrm{K}_{\mathrm{h}_{\mathrm{i}}}^{*} \leq \mathrm{q}$, for $t \in\left(t_{i}, s_{i}\right]$.

(iii) $\left\|\Phi_{\mathrm{i}}^{1} \mathrm{u}(\mathrm{t})+\Phi_{\mathrm{i}}^{2} \mathrm{u}(\mathrm{t})\right\| \leq \mathrm{M}_{1}\left[\mathrm{~K}_{\mathrm{h}_{\mathrm{i}}}\left(\mathrm{D}_{1}^{*} \mathrm{q}+\mathrm{c}_{\mathrm{n}}\right)+\mathrm{K}_{\mathrm{h}_{\mathrm{i}}}^{*}\right]+$ $\left(\mathrm{M}_{0}\left(1+\mathrm{M}_{1}\right)+\frac{\mathrm{M}_{1-\beta}\left(\mathrm{t}_{i+1}-\mathrm{s}_{\mathrm{i}}\right)^{\beta}}{\beta}\right)\left[\left(\mathrm{K}_{\mathrm{G}_{1}}+\widetilde{\mathrm{K}}_{\mathrm{G}_{1}} \mathrm{TKe}_{1}\right) \mathrm{D}_{1}^{*} \mathrm{q}+\right.$ $\left.\mathrm{p}_{1}\right]+\mathrm{M}_{1}\left(\mathrm{t}_{\mathrm{i}+1}-\mathrm{s}_{\mathrm{i}}\right) \mathrm{m}_{\mathrm{G}_{2}}(\mathrm{~s}) \Omega_{\mathrm{G}_{2}}\left[\left(1+\mathrm{TKe}_{2}\right) \mathrm{D}_{1}^{*} \mathrm{q}+\right.$ $\left.\mathrm{p}_{2}\right]+\mathrm{M}_{0} \mathrm{M}_{1}\left(\mathrm{t}_{\mathrm{i}+1}-\mathrm{s}_{\mathrm{i}}\right)\left[\left(\mathrm{K}_{\mathrm{G}_{3}}+\widetilde{\mathrm{K}}_{\mathrm{G}_{3}} \mathrm{TKe}_{3}\right) \mathrm{D}_{1}^{*} \mathrm{q}+\mathrm{p}_{3}\right] \leq \mathrm{q}$, for $\mathrm{t} \in\left(\mathrm{s}_{\mathrm{i}}, \mathrm{t}_{\mathrm{i}+1}\right]$.

(iv) $\left\|\Phi_{\mathrm{i}}^{1} \mathrm{u}(\mathrm{t})+\Phi_{\mathrm{i}}^{2} \mathrm{u}(\mathrm{t})\right\| \leq \mathrm{M}_{1}\left[\mathrm{~K}_{\mathrm{h}_{\mathrm{i}}}\left(\mathrm{D}_{1}^{*} \mathrm{q}+\mathrm{c}_{\mathrm{n}}\right)+\mathrm{K}_{\mathrm{h}_{\mathrm{i}}}^{*}\right]+$ $\left(\mathrm{M}_{0}\left(1+\mathrm{M}_{1}\right)+\frac{\mathrm{M}_{1-\beta} \mathrm{T}^{\beta}}{\beta}\right)\left[\left(\mathrm{K}_{\mathrm{G}_{1}}+\widetilde{\mathrm{K}}_{\mathrm{G}_{1}} \mathrm{TKe}_{1}\right) \mathrm{D}_{1}^{*} \mathrm{q}+\right.$ $\left.\mathrm{p}_{1}\right] \mathrm{M}_{1} \mathrm{Tm}_{\mathrm{G}_{2}}(\mathrm{~s}) \Omega_{\mathrm{G}_{2}}\left[\left(1+\mathrm{TKe}_{2}\right) \mathrm{D}_{1}^{*} \mathrm{q}+\mathrm{p}_{2}\right]+\mathrm{M}_{0} \mathrm{M}_{1} \mathrm{~T}\left[\left(\mathrm{~K}_{\mathrm{G}_{3}}+\right.\right.$ $\left.\left.\widetilde{\mathrm{K}}_{\mathrm{G}_{3}} \mathrm{TKe}_{3}\right) \mathrm{D}_{1}^{*} \mathrm{q}+\mathrm{p}_{3}\right] \leq \mathrm{q}, \mathrm{t} \in[0, \mathrm{~T}]$, where $\mathrm{p}_{1}, \mathrm{p}_{2}, \mathrm{p}_{3}$ are independent of q. dividing both sides by $q$, we have $\Phi_{\mathrm{i}}^{1} \mathrm{u}(\mathrm{t})+$ $\Phi_{\mathrm{i}}^{2} \mathrm{u}(\mathrm{t}) \in \mathrm{B}_{\mathrm{q}}$.

Step 2: Next we will show that $\Phi^{1}=\sum_{\mathrm{i}=0}^{\mathrm{N}} \Phi_{\mathrm{i}}^{1}$ is a contraction. From the definition of $\phi^{1} \mathrm{u}(\mathrm{t}), \Phi_{\mathrm{i}}^{1} \mathrm{u}(\mathrm{t})$ and the assumption of $\left(\mathrm{H}_{2}\right)$, $\left(\mathrm{H}_{4}\right)$ and $\left(\mathrm{H}_{6}\right)$, we get

(i) $\left\|\Phi_{\mathrm{i}}^{1} \mathrm{u}(\mathrm{t})+\Phi_{\mathrm{i}}^{2} \mathrm{u}(\mathrm{t})\right\| \leq \mathrm{D}_{1}^{*}\left[\left(\mathrm{M}_{0}+\frac{\mathrm{M}_{1-\beta} \mathrm{T}^{\beta}}{\beta}\right)\left(\mathrm{K}_{\mathrm{G}_{1}}+\right.\right.$ $\left.\left.\widetilde{\mathrm{K}}_{\mathrm{G}_{1}} \mathrm{TKe}_{1}\right)+\mathrm{M}_{0} \mathrm{M}_{1} \mathrm{~T}\left[\mathrm{~K}_{\mathrm{G}_{3}}+\widetilde{\mathrm{K}}_{\mathrm{G}_{3}} \mathrm{TKe}_{3}\right]\right]\|\mathrm{u}-\overline{\mathrm{u}}\|_{\mathrm{B}_{\mathrm{h}}^{\prime \prime}}, \mathrm{t} \in\left[0, \mathrm{t}_{1}\right]$. (ii) $\left\|\left(\Phi_{\mathrm{i}}^{1} \mathrm{u}\right)(\mathrm{t})-\left(\Phi_{\mathrm{i}}^{1} \overline{\mathrm{u}}\right)(\mathrm{t})\right\| \leq \mathrm{D}_{1}^{*} \mathrm{~K}_{\mathrm{h}_{\mathrm{i}}}\|\mathrm{u}-\overline{\mathrm{u}}\|_{\mathrm{B}_{\mathrm{h}}^{\prime \prime}}, \mathrm{t} \in\left(\mathrm{t}_{\mathrm{i}}, \mathrm{s}_{\mathrm{i}}\right]$, (iii) $\left\|\left(\Phi_{\mathrm{i}}^{1} \mathrm{u}\right)(\mathrm{t})-\left(\Phi_{\mathrm{i}}^{1} \overline{\mathrm{u}}\right)(\mathrm{t})\right\| \leq \mathrm{D}_{1}^{*}\left[\left\{\mathrm{M}_{1} \mathrm{~K}_{\mathrm{h}_{\mathrm{i}}}+\left(\mathrm{M}_{0}\left(1+\mathrm{M}_{1}\right)+\right.\right.\right.$ $\left.\frac{\mathrm{M}_{1-\beta}\left(\mathrm{t}_{\mathrm{i}+1}-\mathrm{s}_{\mathrm{i}}\right)^{\beta}}{\beta}\right)\left[\mathrm{K}_{\mathrm{G}_{1}}+\widetilde{\mathrm{K}}_{\mathrm{G}_{1}} \mathrm{TKe}_{1}\right]+\mathrm{M}_{0} \mathrm{M}_{1}\left(\mathrm{t}_{\mathrm{i}+1}-\mathrm{s}_{\mathrm{i}}\right) \mathrm{D}_{1}^{*}\left[\mathrm{~K}_{\mathrm{G}_{3}}+\right.$ $\left.\left.\widetilde{\mathrm{K}}_{\mathrm{G}_{3}} \mathrm{TKe}_{3}\right]\right\} \times\|\mathrm{u}-\overline{\mathrm{u}}\|_{\mathrm{B}_{\mathrm{h}}^{\prime \prime}}, \mathrm{t} \in\left(\mathrm{s}_{\mathrm{i}}, \mathrm{t}_{\mathrm{i}+1}\right]$ (iv) $\left\|\left(\Phi_{\mathrm{i}}^{1} \mathrm{u}\right)(\mathrm{t})-\left(\Phi_{\mathrm{i}}^{1} \overline{\mathrm{u}}\right)(\mathrm{t})\right\| \leq \mathrm{D}_{1}^{*}\left\{\mathrm{M}_{1} \mathrm{~K}_{\mathrm{h}_{\mathrm{i}}}+\left(\mathrm{M}_{0}\left(1+\mathrm{M}_{1}\right)+\right.\right.$ $\left.\left.\frac{\mathrm{M}_{1-\beta} \mathrm{T}^{\beta}}{\beta}\right)\left[\mathrm{K}_{\mathrm{G}_{1}}+\widetilde{\mathrm{K}}_{\mathrm{G}_{1}} \mathrm{TKe}_{1}\right]+\mathrm{M}_{0} \mathrm{M}_{1} \mathrm{D}_{1}^{*}\left[\mathrm{~K}_{\mathrm{G}_{3}}+\widetilde{\mathrm{K}}_{\mathrm{G}_{3}} \mathrm{TKe}_{3}\right]\right\} \| \mathrm{u}-$ $\overline{\mathrm{u}}\left\|_{\mathrm{B}_{\mathrm{h}}^{\prime \prime}}, \mathrm{t} \in(0, \mathrm{~T}] \leq \Lambda^{*}\right\| \mathrm{u}-\overline{\mathrm{u}} \|_{\mathrm{B}_{\mathrm{h}}^{\prime \prime}}$, where $\Lambda^{*}=\mathrm{D}_{1}^{*}\left\{\mathrm{M}_{1} \mathrm{~K}_{\mathrm{h}_{\mathrm{i}}}+\right.$ $\left(\mathrm{M}_{0}\left(1+\mathrm{M}_{1}\right)+\frac{\mathrm{M}_{1-\beta} \mathrm{T}^{\beta}}{\beta}\right)\left[\mathrm{K}_{\mathrm{G}_{1}}+\widetilde{\mathrm{K}}_{\mathrm{G}_{1}} \mathrm{TKe}_{1}\right]+\mathrm{M}_{0} \mathrm{M}_{1} \mathrm{D}_{1}^{*}\left[\mathrm{~K}_{\mathrm{G}_{3}}+\right.$ $\left.\left.\widetilde{\mathrm{K}}_{\mathrm{G}_{3}} \mathrm{TKe}_{3}\right]\right\}<1 . \phi^{1} \mathrm{u}(\mathrm{t})$ is a contraction.

Step 3: Next we will prove that $\Phi_{\mathrm{i}}^{2}$ is compact and continuous. We split the proof into three parts

Let the sequence $u_{n}$ such that $u_{n} \rightarrow u$ in $B_{h}^{\prime \prime}$. Then for all $t \in \mathrm{I}$ by the definition of $\Phi^{2} \mathrm{u}(\mathrm{t}), \Phi_{\mathrm{i}}^{2} \mathrm{u}(\mathrm{t})$ and by assumptions $\mathrm{H}(3)$ and $\mathrm{H}(6)$

$\left\|\left(\Phi_{\mathrm{i}}^{2} \mathrm{u}^{\mathrm{n}}\right)(\mathrm{t})-\left(\Phi_{\mathrm{i}}^{2} \mathrm{u}\right)(\mathrm{t})\right\| \leq M_{1} t_{1} \| \mathrm{G}_{2}\left(\mathrm{~s}, \mathrm{u}_{\mathrm{s}}^{\mathrm{n}}+\mathrm{v}_{\mathrm{s}}, \int_{0}^{\mathrm{s}} \mathrm{e}_{2}(\mathrm{~s}, \tau\right.$, $\left.u_{\tau}^{n}+v_{\tau}\right) d \tau-G_{2}\left(s, u_{s}+v_{s}, \int_{0}^{s} e_{2}\left(s, \tau, u_{\tau}+v_{\tau}\right) d \tau \|, t \in\left[0, t_{1}\right]\right.$ $\left\|\left(\Phi_{\mathrm{i}}^{2} \mathrm{u}^{\mathrm{n}}\right)(\mathrm{t})-\left(\Phi_{\mathrm{i}}^{2} \mathrm{u}\right)(\mathrm{t})\right\| \leq M_{1}\left(t_{i+1}-s_{i}\right) \| \mathrm{G}_{2}\left(\mathrm{~s}, \mathrm{u}_{\mathrm{s}}^{\mathrm{n}}+\right.$ $\mathrm{v}_{\mathrm{s}}, \int_{0}^{\mathrm{s}} \mathrm{e}_{2}\left(\mathrm{~s}, \tau, \mathrm{u}_{\tau}^{\mathrm{n}}+\mathrm{v}_{\tau}\right) \mathrm{d} \tau-\mathrm{G}_{2}\left(\mathrm{~s}, \mathrm{u}_{\mathrm{s}}+\mathrm{v}_{\mathrm{s}}, \int_{0}^{\mathrm{s}} \mathrm{e}_{2}\left(\mathrm{~s}, \tau, \mathrm{u}_{\tau}+\right.\right.$ $\left.\mathrm{v}_{\tau}\right) \mathrm{d} \tau \|, \mathrm{t} \in\left[s_{i}, t_{i+1}\right]$ and $\mathrm{G}_{2}, \mathrm{G}_{3}$ are continuous, we know that $\left\|\left(\Phi_{\mathrm{i}}^{2} \mathrm{u}^{\mathrm{n}}\right)(\mathrm{t})-\left(\Phi_{\mathrm{i}}^{2} \mathrm{u}\right)(\mathrm{t})\right\| \rightarrow 0$ as $\mathrm{n} \rightarrow \infty, \mathrm{u}_{\mathrm{n}} \rightarrow \mathrm{u}$ which shows that $\Phi^{2}$ is continuous.

(b) $\Phi^{2}$ maps bounded sets into bounded sets in $\mathrm{B}_{\mathrm{h}}^{\prime \prime}$

It is sufficient to demonstrate that for any $\mathrm{R}>0$,there exists $\mathrm{R}^{\prime}>0$ such that for each $\mathrm{u} \in \mathrm{B}_{\mathrm{q}}=\mathrm{u} \in \mathrm{B}_{\mathrm{h}}{ }^{\prime \prime}:\|\mathrm{u}\|_{\mathrm{PC}} \leq \mathrm{R}$, we have $\left\|\Phi^{2} \mathrm{u}\right\|_{\mathrm{B}_{\mathrm{h}}}{ }^{\prime \prime} \leq \mathrm{R}^{\prime}$. From the definition of $\Phi^{2} \mathrm{u}(\mathrm{t})$,

$\left\|\left(\Phi^{2}{ }_{i} \mathrm{u}\right)(\mathrm{t})\right\| \leq \mathrm{M}_{1} \mathrm{t}_{1} \mathrm{~m}_{\mathrm{G}_{2}}(\mathrm{~s}) \Omega_{\mathrm{G}_{2}}\left[\left(1+\mathrm{K}_{\mathrm{e}_{2}}\right) \mathrm{D}_{1^{*}} \mathrm{Q}+\mathrm{p}_{2}\right], \mathrm{t} \in\left[\mathrm{o}, \mathrm{t}_{1}\right]$, $\left\|\left(\Phi{ }^{2} \mathrm{u}\right)(\mathrm{t})\right\| \leq \mathrm{M}_{1}\left(\mathrm{t}_{\mathrm{i}+1}-\mathrm{s}_{\mathrm{i}}\right) \mathrm{m}_{\mathrm{G}_{2}}(\mathrm{~s}) \Omega_{\mathrm{G}_{2}}\left[\left(1+\mathrm{K}_{\mathrm{e}_{2}}\right) \mathrm{D}_{1^{*}} \mathrm{Q}+\mathrm{p}_{2}\right]$, $\mathrm{t} \in\left[\mathrm{s}_{\mathrm{i}}, \mathrm{t}_{\mathrm{i}+1}\right]$,

$\left\|\left(\Phi{ }^{2} \mathrm{u}\right)(\mathrm{t})\right\| \leq \mathrm{M}_{1} \operatorname{Tm}_{\mathrm{G}_{2}}(\mathrm{~s}) \Omega_{\mathrm{G}_{2}} \quad\left[\left(1+\mathrm{K}_{\mathrm{e}_{2}}\right) \mathrm{D}_{1^{*}} \mathrm{Q}+\mathrm{p}_{2}\right] \leq \mathrm{R}, \mathrm{t} \in$ $[0, \mathrm{~T}]$.

Then we conclude that $\Phi^{2}$ maps bounded sets into bounded sets. (c)Finally, we exhibit that $\Phi^{2}$ maps bounded sets into equicontinuous set.

For, interval $t \in\left[s_{i}, t_{i+1}\right], s_{i} \leq l_{1} \leq l_{2} \leq t_{i+1}, i=1, \ldots, N$, for every $\mathrm{u}(\mathrm{t}) \in \mathrm{B}_{\mathrm{q}}$, by definition of $\left(\Phi^{2}{ }_{\mathrm{i}} \mathrm{u}\right)(\mathrm{t})$ and hypotheses $\mathrm{H}(3)$ and $\mathrm{H}(6)$,

$\left.\|\left(\Phi^{2}{ }_{\mathrm{i}} \mathrm{u}\right)\left(\mathrm{l}_{2}\right)-\Phi^{2}{ }_{\mathrm{i}} \mathrm{u}\right)\left(\mathrm{l}_{1}\right) \| \leq \mathrm{M}_{1}\left(\mathrm{l}_{2}-\mathrm{l}_{1}\right) \mathrm{m}_{\mathrm{G}_{2}}(\mathrm{~s}) \Omega_{\mathrm{G}_{2}}[(1+$ $\left.\mathrm{Ke}_{2} \mathrm{D}_{1}^{*} \mathrm{q}+\mathrm{p}_{2}\right]+\left(\mathrm{l}_{1}-\mathrm{s}_{\mathrm{i}}\right)\left\|\mathrm{T}\left(\mathrm{l}_{2}-\mathrm{s}\right)-\mathrm{T}\left(\mathrm{l}_{1}-\mathrm{s}\right)\right\| \mathrm{m}_{\mathrm{G}_{2}}(\mathrm{~s}) \Omega_{\mathrm{G}_{2}}$ $+\left[\left(1+K_{e_{2}}\right) D_{1}^{*} q+p_{2}\right], t \in\left[s_{i}, t_{i+1}\right]$, as $l_{1} \rightarrow l_{2}$ the right hand side tends to zero is equicontinuous.

Step 4: $\Phi_{\mathrm{i}}^{2}$ maps $\mathrm{B}_{\mathrm{q}}$ into a precompact set in $B_{h}^{\prime \prime}$.

Now, we shall prove that $\Phi_{i}^{2}$ is relatively compact in $\Phi_{i}^{2}$. Obiviously $\Phi_{\mathrm{i}}^{2}$ is relatively compact in $\mathrm{B}_{\mathrm{h}}^{\prime \prime}$ for $\mathrm{t}=0,0<\varepsilon<t$, for $\mathrm{u} \in \mathrm{B}_{\mathrm{q}}$. We define

$\left(\Phi_{\mathrm{i}}^{2, \varepsilon} \mathrm{u}\right)(\mathrm{t})=\mathrm{T}(\varepsilon) \int_{0}^{\mathrm{t}-\varepsilon} \mathrm{T}(t-s-\varepsilon) \mathrm{G}_{2}\left(\mathrm{~s}, \mathrm{u}_{\mathrm{s}}+\mathrm{v}_{\mathrm{s}}, \int_{0}^{\mathrm{s}} \mathrm{e}_{2}\left(\mathrm{~s}, \tau, \mathrm{u}_{\tau}+\right.\right.$ $\left.\mathrm{v}_{\tau}\right) \mathrm{d} \tau \mathrm{ds}$.

For the reason that $\mathrm{T}(\mathrm{t})$ is compact operator, $\mathrm{V}_{\varepsilon}(\mathrm{t})=$ $\left\{\left(\Phi_{\mathrm{i}}^{2, \varepsilon} \mathrm{u}\right)(\mathrm{t}): \mathrm{u} \in \mathrm{B}_{\mathrm{q}}\right\}$ is relatively compact in $\mathrm{X}$ for every is relatively compact in $\mathrm{X}$ for every $\varepsilon$, for every $0<\varepsilon<\mathrm{t}$, for each $\mathrm{u} \in \mathrm{B}_{\mathrm{q}}$,

$$
\begin{aligned}
\left\|\left(\Phi_{\mathrm{i}}^{2} \mathrm{u}\right)(\mathrm{t})-\left(\Phi_{\mathrm{i}}^{2, \varepsilon} \mathrm{u}\right)(\mathrm{t})\right\| \\
\quad \leq \int_{\mathrm{t}-\varepsilon}^{\mathrm{t}} \mathrm{M}_{1} \mathrm{~m}_{\mathrm{G}_{2}}(\mathrm{~s}) \Omega_{\mathrm{G}_{2}}\left[\left(1+\mathrm{K}_{\mathrm{e}_{2}}\right) \mathrm{D}_{1}^{*} \mathrm{q}+\mathrm{p}_{2}\right] \mathrm{ds} \\
\leq \epsilon[\Lambda] \rightarrow 0 \text { as } \epsilon \rightarrow 0 .
\end{aligned}
$$

which are relatively compact sets arbitrarily close to the set $V_{\epsilon}(t), t>0$ as a result $V_{\epsilon}(t)$ is relatively compact in $X$. From the above steps, it follows by the Krasnoselskii's fixed point theorem, we get that $\bar{\Phi}$ has at least one fixed point $\mathrm{u}(\mathrm{t}) \in \mathrm{B}_{\mathrm{h}}^{\prime \prime}$. With these, a fixed point of the operator $\bar{\Phi}$ is the mild solution $u$ of the problem (1) - (3). This finishes the verification of the hypothesis. 


\section{Applications}

To epitomize our hypothetical results, now, we consider the following INIDE with infinite delay of the structure

$$
\begin{aligned}
& \frac{\partial}{\partial t}\left[u ( t , x ) \left\{\int_{-\infty}^{t} a_{1}(t, x, s-t) P_{1}(u(s, x)) d s+\right.\right. \\
& \left.\left.\quad \int_{0}^{t} \int_{-\infty}^{s} K_{1}(s, \tau) P_{2}(u(\tau, x)) d \tau d s\right\}\right]=\frac{\partial^{2}}{\partial x^{2}} u(t, x)\left\{\int_{-\infty}^{t} a_{2}(t, x, s-\right. \\
& t) Q_{1}(u(s, x)) d s+\int_{0}^{t} \int_{-\infty}^{s} K_{2}(s, \tau) Q_{2}(u(\tau, x)) d \tau d s+ \\
& \left\{\int_{-\infty}^{t} a_{3}(t, x, s-t) Q_{3}(u(s, x)) d s+\right. \\
& \left.\left.\int_{0}^{t} \int_{-\infty}^{s} K_{3}(s, \tau) Q_{4}(u(\tau, x)) d \tau d s\right\}\right\}, X \in[0, \pi], t \in[0, b], t \neq t_{k},
\end{aligned}
$$

$\mathrm{u}(\mathrm{t}, 0)=0=\mathrm{u}(\mathrm{t}, \pi)=0, \mathrm{t} \geq 0$

$\mathrm{u}(\mathrm{t}, \mathrm{x})=\varphi(\mathrm{t}, \mathrm{x}), \mathrm{t} \in(-\infty, 0], \mathrm{x} \in[0, \pi]$,

$u_{i}(t, x)=\int_{-\infty}^{t} \eta\left(t_{i}-s\right) u(s, x) d s,(t, x) \in\left(t_{i}, s_{i}\right] \times[0, \pi]$,

The prefixed real numbers are $0<\mathrm{t}_{1}<\mathrm{t}_{2}<\cdots<\mathrm{t}_{\mathrm{n}}<b$ and $\varphi \in \mathrm{B}_{\mathrm{h}}$. Let $\mathrm{X}=\mathrm{L}^{2}[0, \pi]$ whose norm is $|\cdot|_{\mathrm{L}^{2}}$ and determine the operator $\mathrm{A}: \mathrm{D}(\mathrm{A}) \subset \mathrm{X} \rightarrow \mathrm{X}$ by $\mathrm{Aw}=\mathrm{w}^{\text {" with the domain }}$ $\mathrm{D}(\mathrm{A})=\left\{\mathrm{wEX}: \mathrm{w}, \mathrm{w}^{\prime}\right.$ are absolutely continuous, $\mathrm{w}^{\prime \prime} \in$ $\mathrm{X}, \mathrm{w}(0)=\mathrm{w}(\pi)=0\}$. Then Aw $=\sum_{\mathrm{n}=1}^{\infty} \mathrm{n}^{2}\left\langle\mathrm{w}, \mathrm{w}_{\mathrm{n}}\right\rangle \mathrm{w}_{\mathrm{n}}, \mathrm{w} \in$ $\mathrm{D}(\mathrm{A})$, in which $\mathrm{w}_{\mathrm{n}}(\mathrm{s})=\sqrt{\left(\frac{2}{\pi}\right)} \sin (\mathrm{ns}), \mathrm{n}=1,2, \ldots$,

is the orthogonal set of eigenvectors of $A$. $T(t) w=$ $\sum_{\mathrm{n}=1}^{\infty} \mathrm{e}^{-\mathrm{n}^{2} \mathrm{t}}\left\langle\mathrm{w}, \mathrm{w}_{\mathrm{n}}\right\rangle \mathrm{w}_{\mathrm{n}}$, for all $\mathrm{w} \in \mathrm{X}$, and every $\mathrm{t}>0$. For each $\mathrm{w} \in \mathrm{X},(\mathrm{A})^{\frac{1}{2}} \mathrm{~W}=\sum_{\mathrm{n}=1}^{\infty}(\mathrm{n})\left\langle\mathrm{w}, \mathrm{w}_{\mathrm{n}}\right\rangle \mathrm{w}_{\mathrm{n}} \quad$ and $\quad\left\|(A)^{\frac{1}{2}}\right\|=1$, $\mathrm{g}\left([0, \mathrm{~b}] \times \mathrm{B}_{\mathrm{h}} \times \mathrm{L}^{2}\right) \subseteq \mathrm{D}(-\mathrm{A})^{\frac{1}{2}}$,

$\left\|(\mathrm{A})^{\frac{1}{2}} \mathrm{G}_{1}\left(\mathrm{t}, \varphi_{1}, \mu_{1}\right) \mathrm{x}-(\mathrm{A})^{\frac{1}{2}} \mathrm{G}_{1}\left(\mathrm{t}, \varphi_{2}, \mu_{2}\right) \mathrm{x} \leq \mathrm{N}_{1}\left[\| \varphi_{1}-\varphi_{2}\right]\right\|_{\mathrm{B}_{\mathrm{h}}}+$ $\left.\left|\mu_{1}-\mu_{2}\right|\right]$ for $N_{1}>0$ and

$\left\|(A)^{\frac{1}{2}} G_{3}\left(t, \varphi_{1}, \mu_{1}\right) x-(A)^{\frac{1}{2}} G_{3}\left(t, \varphi_{2}, \mu_{2}\right) x \leq N_{2}\left[\| \varphi_{1}-\varphi_{2}\right]\right\|_{B_{h}}+$ $\left.\left|\mu_{1}-\mu_{2}\right|\right]$ for $\mathrm{N}_{2}>0$.

The continuous functions $Q_{i}, i=1,2$ are defines for each $(\theta, x) \in$ $(-\infty, 0] \times[0, \pi]$, and $0 \leq \mathrm{Q}_{\mathrm{i}}(\mathrm{u}(\theta)(\mathrm{x})) \leq \prod\left(\int_{-\infty}^{0} \mathrm{e}^{2 \mathrm{~s}}\|\mathrm{u}(\mathrm{s}, .)\|_{\mathrm{L}^{2}} \mathrm{ds}\right)$ and the continuous non decreasing function is defined as $\Pi:[0, \infty] \rightarrow(0, \infty)$ and we can take $\Omega_{\mathrm{G}_{2}}(\mathrm{r})=\Pi(\mathrm{r})$ in (H3). Presently we can see that

$$
\begin{gathered}
\left\|\mathrm{G}_{2}\left(\mathrm{t}, \varphi, \mathrm{H}_{2} \varphi\right)\right\|_{\mathrm{L}^{2}}=\left[\int _ { 0 } ^ { \pi } \left(\int_{-\infty}^{0} \mathrm{a}_{2}(\mathrm{t}, \mathrm{x}, \theta) \mathrm{Q}_{1}(\varphi(\theta))(\mathrm{x}) \mathrm{d} \theta\right.\right. \\
\left.\left.+\left(\mathrm{H}_{2} \varphi(\theta)\right)(\mathrm{x})\right)^{2} \mathrm{dx}\right]^{\frac{1}{2}} \\
\leq\left\{\left[\int_{0}^{\pi}\left(\mathrm{m}_{1}(\mathrm{t}, \mathrm{x})\right)^{2} \mathrm{dy}\right]^{\frac{1}{2}}+\left[\int_{0}^{\pi}\left(\mathrm{m}_{2}(\mathrm{t})\right)^{\frac{1}{2}} \mathrm{dx}\right]^{\frac{1}{2}}\right\} \Pi\left(\|\varphi\|_{h}\right) \\
\leq\left[\overline{\mathrm{m}_{1}}(\mathrm{t})+\sqrt{\pi} \overline{\mathrm{m}_{2}}(\mathrm{t})\right] \Pi\left(\|\varphi\|_{h}\right) \leq \mathrm{m}(\mathrm{t})\left(\|\varphi\|_{h}\right)
\end{gathered}
$$

Since $\Pi:[0, \infty] \rightarrow(0, \infty)$ is a continuous and non decreasing function, We can take $\mathrm{m}(\mathrm{t})=\left\{\overline{\mathrm{m}_{1}}(\mathrm{t})+\sqrt{\pi} \overline{\mathrm{m}_{2}}(\mathrm{t})\right\}$. Along these lines the condition (H3) holds. Hence by theorem 3.1, we comprehend that the system (8)-(11) has a unique mild solution on I.

\section{References}

[1] Herandez E \& O'Regan D (2013), On a new class of abstract impulsive differential equations, Proceedings of the American Mathematical Society, 141, 1641-1649.

[2] Pazy A (1983), Semigroups of Linear Operators and Applications to Partial Differential Equations, Springer-Verlag, New York.

[3] Pierri M, O'Regan D \& Rolnik V (2013), Existence of solutions for semi-linear abstract differential equations with not instantaneous impulses, Applied Mathematics and Computation, 219, 6743-6749.
[4] Nadeem M \& Dabas J (2016), Existence Results for Fractional Stochastic Differential Equation with Impulsive Effect, International Journal of Nonlinear Science, 22, 131-139. 